\title{
The geography of HIVIAIDS prevalence rates in Botswana
}

\author{
This article was published in the following Dove Press journal: \\ HIVIAIDS - Research and Palliative Care \\ 17 July 2012 \\ Number of times this article has been viewed
}

\section{Ngianga-Bakwin Kandala' \\ Eugene K Campbell ${ }^{2}$ \\ Serai Dan Rakgoasi ${ }^{2}$ \\ Banyana C Madi-Segwagwe ${ }^{3}$ \\ Thabo T Fako ${ }^{4}$}

'University of Warwick, Warwick

Medical School, Division of

Health Sciences; Populations,

Evidence and Technologies Group,

Warwick Evidence, Coventry, UK;

${ }^{2}$ Department of Population Studies,

University of Botswana, ${ }^{3}$ SADC

Secretariat, Directorate of Social

and Human Development and Special

Programmes, ${ }^{4}$ Vice Chancellor's

Office, University of Botswana,

Gaborone, Botswana
Correspondence: Ngianga-Bakwin Kandala, University of Warwick, Warwick Medical School, Division of Health Sciences, Gibbet Hill Road, CV4 7AL, Coventry, UK

Tel +44247 6I5 054I

Email n-b.kandala@warwick.ac.uk
Background: Botswana has the second-highest human immunodeficiency virus (HIV) infection rate in the world, with one in three adults infected. However, there is significant geographic variation at the district level and HIV prevalence is heterogeneous with the highest prevalence recorded in Selebi-Phikwe and North East. There is a lack of age-and location-adjusted prevalence maps that could be used for targeting HIV educational programs and efficient allocation of resources to higher risk groups.

Methods: We used a nationally representative household survey to investigate and explain district level inequalities in HIV rates. A Bayesian geoadditive mixed model based on Markov Chain Monte Carlo techniques was applied to map the geographic distribution of HIV prevalence in the 26 districts, accounting simultaneously for individual, household, and area factors using the 2008 Botswana HIV Impact Survey.

Results: Overall, HIV prevalence was $17.6 \%$, which was higher among females $(20.4 \%)$ than males $(14.3 \%)$. HIV prevalence was higher in cities and towns $(20.3 \%)$ than in urban villages and rural areas (16.6\% and $16.9 \%$, respectively). We also observed an inverse U-shape association between age and prevalence of HIV, which had a different pattern in males and females. HIV prevalence was lowest among those aged 24 years or less and HIV affected over a third of those aged 25-35 years, before reaching a peak among the 36-49-year age group, after which the rate of HIV infection decreased by more than half among those aged 50 years and over. In a multivariate analysis, there was a statistically significant higher likelihood of HIV among females compared with males, and in clerical workers compared with professionals. The district-specific net spatial effects of HIV indicated a significantly higher HIV rate of $66 \%$ (posterior odds ratio of 1.66) in the northeast districts (Selebi-Phikwe, Sowa, and Francistown) and a reduced rate of $27 \%$ (posterior odds ratio of 0.73 ) in Kgalagadi North and Kweneng West districts.

Conclusion: This study showed a clear geographic distribution of the HIV epidemic, with the highest prevalence in the east-central districts. This study provides age- and location-adjusted prevalence maps that could be used for the targeting of HIV educational programs and efficient allocation of resources to higher risk groups. There is need for further research to determine the social, cultural, economic, behavioral, and other distal factors that might explain the high infection rates in some of the high-risk areas in Botswana.

Keywords: Botswana, HIV prevalence, geographic location, spatial autocorrelation

\section{Introduction}

The emergence and alarming spread of the human immunodeficiency virus (HIV) and acquired immune deficiency syndrome (AIDS) since the early 1980s have caused considerable concern about sexual and reproductive health among populations throughout 
the world, especially sub-Saharan Africa, which is home to 70 percent of the world's HIV-infected people. ${ }^{1}$

Southern Africa, particularly the Southern African Development Community, is the most affected region worldwide. ${ }^{1}$ Botswana has the second-highest HIV infection rate in the world after Swaziland, ${ }^{1}$ with one in three adults infected. In 2007-2009, the HIV prevalence among males and females aged 15-49 years in east, west, and central Africa was $2.1 \%$ and $3.0 \%, 4.5 \%$ and $6.7 \%$, and $1.9 \%$ and $3.1 \%$, respectively. ${ }^{2}$ During the same period, HIV prevalence among males and females in Botswana was $18.9 \%$ and $28.9 \%$, respectively. ${ }^{2}$ Botswana's HIV/AIDS epidemic is substantial in magnitude and impact; for example, $12 \%$ of children in Botswana have been orphaned due to AIDS. ${ }^{1}$

While the impact of HIV/AIDS has been clear for all to see, estimating the population-based HIV prevalence rate has been a challenge for a number of years due to an absence of data. Subsequently, most estimates of HIV prevalence relied heavily on data derived from sentinel surveillance of pregnant women attending antenatal care.

Current estimates from the Department of HIV/AIDS Prevention and Care of HIV prevalence among pregnant women aged 15-49 years attending antenatal care in public health clinics in Botswana was 31.8\%. ${ }^{3}$ The national HIV prevalence amongst the women surveyed showed a decline in prevalence from $36.2 \%$ in 2001 to $31.8 \%$ in 2009 . However, there is significant geographic variation at the district level, and HIV prevalence is heterogeneous in Botswana's districts, with the highest prevalence recorded in Selebi-Phikwe and North East (41.6\%). This is followed by Tutume (41.1\%), Bobirwa (39.6\%), and Chobe (39.3\%). Hukuntsi has the lowest prevalence rate (16.1\%). These HIV prevalence rates and those reported in the 2008 Botswana AIDS Impact Survey (BAIS III) $^{3}$ followed similar patterns, but mask significant variations within the population. Meanwhile, populationlevel socioeconomic and health resource characteristics have explained little of the variation in rates.

The success of any HIV/AIDS policy intervention depends on a broad and accurate understanding of the socioeconomic, environmental, and cultural factors that determine the spread of the disease. Until recently, available information on HIV prevalence was derived from antenatal clinics. However, information obtained from antenatal clinics represents only a small proportion of all cases in the general population, since many other cases do not seek medical attention in antenatal clinics. Thus, the antenatal clinics data may not be appropriate for estimating the prevalence of HIV for program developments.
Policy makers often want to know the distribution of HIV prevalence by geographical region, or association with environmental and cultural factors. In this regard, mapping risk variations in HIV rates is an invaluable tool. Further, the mapping of variation in risk of HIV rates can help improve the targeting of scarce resources for public health interventions.

The Geographic Information System (GIS) ${ }^{4}$ is, therefore, a powerful tool for public health practitioners that easily aids assessment of patterns, trends, and relationships between health events and environmental, cultural, socioeconomic, and other geographic factors. GIS further helps us to understand HIV rates at the community level and assists with identifying underserved populations. It can also help public health agencies to efficiently allocate scarce program resources to appropriate locations, assist in identifying at-risk populations, and determine where to focus efforts to prevent HIV.

The benefits of using geostatistical methods combined with GIS are largely unknown and have not been investigated using the BAIS III data. This paper reports the results obtained using a nationally representative household survey by exploring the spatial distribution of HIV rates in Botswana, taking into account district-level factors. The BAIS III is the most recent AIDS impact survey conducted in Botswana and is a valuable resource for population-based HIV prevalence data.

To gain more understanding of the geographic variation or patterns based on the observed HIV rates, a Bayesian hierarchical model was fitted, with the inclusion of spatial (district), nonlinear metrical (age at HIV diagnosis) covariates, and other confounding risk factors such as employment. Of particular interest in this study was whether a significant geographic variation in HIV rates as observed in previous data existed, and, if so, what potential risk factors could explain such variation, taking into account population mobility and localized effects.

\section{Methods}

\section{Data collection}

The BAIS III is the third and latest of a series of nationally representative demographic surveys, conducted by the Botswana Central Statistical Office and funded by the Government of Botswana. Its aim was also to provide up-to-date information on the country's HIV and AIDS epidemic.

The 2001 Botswana Population Housing Census ${ }^{3}$ provided the sample frame for BAIS III, and ethical approval was obtained from the Botswana Ministry of Health. Overall, 16,992 eligible respondents aged 10-64 years (males and females) 
were identified from 7600 households, of whom 15,878 were successfully interviewed, yielding an individual response rate of $93 \%$. For this study we did not consider ethical approval relevant because it was a secondary analysis of data. The sample population was treated anonymously and it was impossible to identify particular individuals.

\section{Statistical analysis}

We applied a Bayesian geoadditive semiparametric mixed model, a unified approach that explores geographic patterns in the prevalence of HIV infection and possible nonlinear effects within a simultaneous, coherent Bayesian regression framework. The model employed a fully Bayesian approach using Markov Chain Monte Carlo (MCMC) techniques for inference and model-checking. ${ }^{5}$ The model and statistical techniques utilized have been described in more detail elsewhere. ${ }^{6,7}$ The model is implemented in the BayesX (version 2.0.1; University of Munich, Munich, Germany) software package, which permits Bayesian inference based on MCMC simulation techniques. The statistical significance of apparent associations between potential risk factors and the prevalence of HIV was explored in chi-square and Mann-Whitney $U$-tests, as appropriate. A $P$-value of $<0.05$ was considered indicative of a statistically significant difference.

In the multivariate analysis, we evaluated the significance of the posterior odds ratio (POR) for the fixed, nonlinear, and spatial effects using the deviance information criterion as a measure of fit and model complexity. To account for possible departures from the assumed distribution, 95\% credible regions for the PORs and probability maps (the equivalent of confidence intervals for the spatial effects) were calculated using robust standard errors estimated via MCMC simulation techniques. Although this estimation process is used less frequently in the literature, the estimated PORs that were produced could be interpreted as similar to those of ordinary logistic models.

\section{Results}

Overall, HIV prevalence was $17.6 \%$, which was higher among females $(20.4 \%)$ than males $(14.3 \%)$. HIV prevalence was higher in cities and towns $(20.3 \%)$ than in urban villages and rural areas (16.6\% and $16.9 \%$, respectively) (Table 1$)$. We also observed an inverse U-shape association between age and the prevalence of HIV. HIV prevalence was lowest among those aged 24 years or less $(5.6 \%)$ and reached over a third $(33.2 \%)$ in those in the $25-35$ year age group, before reaching a peak (37.1\%) among those aged 36-49 years and then declining by more than half $(16.9 \%)$ in those aged 50 years and over. The mean age of males was lower compared to females (25.1 years, standard deviation [SD] 19.3 years versus 27.2 years, SD 20.5 years) (Table 1 ).

The unadjusted marginal ORs indicated that in 2008 the highest HIV prevalence was in Selebi-Phikwe followed by Sowa and Francistown. The lowest prevalence of HIV was observed in Kgalagadi North and Kweneng West districts. The districts were also ranked according to their respective HIV prevalence rates and the corresponding marginal odds ratios (ORs) (Figure 1).

The pattern of HIV prevalence did not differ markedly between districts in terms of marginal ORs and the prevalence rates, with consistently higher prevalence in SelebiPhikwe, Sowa, and Francistown, and lower prevalence in Kweneng West district (Table 1). The prevalence rates and marginal ORs of HIV at the district level indicated that 15 districts, namely Gaborone, Lobatse, Jwaneng, Ngwaketse West, Kweneng East, Central-Boteti, Kgatleng, Southern, Barolong, Ghanzi, Southeast, Kgatleng, Ngamiland North, Kgalagadi North, and Kweneng West, had HIV prevalence rates that were below the national prevalence (Table 1).

The results of the multivariate analysis using Bayesian geoadditive regression analyses indicate that HIV rates were higher among females than males and among clerical jobholders than professionals, which are consistent with the unadjusted results (Table 1). The district-specific net spatial effects of HIV, which include the total residual spatial effects of the district (ie, the sum of both the structured and unstructured spatial effects; see Table 1) indicated a statistically significant higher HIV rate of $66 \%$ (POR of 1.66) in the northeast districts (Selebi-Phikwe, Sowa, and Francistown) and a reduced rate of $27 \%$ (POR of 0.73 ) in Kgalagadi North and Kweneng West districts (Figure 2). Two important observations emerged. First, after accounting for spatial dependencies of districts in the data, the districts with the highest HIV prevalence in the North East now included only Francistown and Selebi-Phikwe (not Sowa) while Gaborone remained the lowest prevalence district as suggested by the marginal OR (Table 1). Second, there was a strong northeast-south gradient in these district effects, with a fairly sharp dividing-line running through the central districts. Over and above the impact of the fixed effects, there appeared to be a higher risk of HIV in the northeastern districts (North East, including Francistown, and Central Bobonong, including Selebi-Phikwe). This was quite general and affected most of the northeastern districts. Moreover, age 
Table I Prevalence and crude marginal odds ratio of HIV infection* by baseline characteristics ${ }^{3}$

\begin{tabular}{|c|c|c|c|c|}
\hline \multirow[t]{2}{*}{ Variables } & \multicolumn{4}{|c|}{ HIV BAIS III 2008: I4,344 } \\
\hline & Cases, n, (\%) & $P$-value** & Marginal OR and $95 \% \mathrm{Cl}^{* * * *}$ & Posterior OR and $95 \% \mathrm{Cl}^{\dagger}$ \\
\hline Number & $252 I(I 7.6)$ & & & \\
\hline \multicolumn{5}{|l|}{ Sex } \\
\hline Male & $933(14.3)$ & & & 1.00 \\
\hline Female & I588 (20.3) & $<0.001$ & & $1.50(1.31,1.67)$ \\
\hline \multicolumn{5}{|l|}{ Place of residence } \\
\hline Cities/towns & $643(20.3)$ & & & I.08 $(0.88,1.29)$ \\
\hline Urban villages & $778(16.6)$ & & & $1.08(0.93,1.29)$ \\
\hline Rural areas & $1100(16.9)$ & $<0.001$ & & 1.00 \\
\hline \multicolumn{5}{|l|}{ Age at diagnosis } \\
\hline$\leq 24$ years & $405(5.6)$ & & & See Figure 3 \\
\hline $25-35$ years & $969(33.2)$ & & & \\
\hline $36-49$ years & $799(37.1)$ & & & \\
\hline $50+$ years & $348(16.9)$ & $<0.001$ & & \\
\hline \multicolumn{5}{|l|}{ Occupation } \\
\hline Professional & $199(20.7)$ & & & 1.00 \\
\hline Clerical $/$ manual & $976(28.7)$ & $<0.001$ & & $1.87(1.55,2.27)$ \\
\hline \multicolumn{5}{|c|}{ District of residence ${ }^{* * *}$} \\
\hline Gaborone & $248(16.7)$ & & $1.73(1.17,2.56)$ & See Figure 2A \\
\hline Francistown & $169(24.2)$ & & $2.75(1.83,4.12)$ & \\
\hline Lobatse & $38(16.7)$ & & $1.73(1.05,2.87)$ & \\
\hline Selebi-Phikwe & $143(27.6)$ & & $3.29(2.17,4.97)$ & \\
\hline Orapa & $13(17.8)$ & & $1.87(0.93,3.77)$ & \\
\hline Jwaneng & $14(16.1)$ & & $1.65(0.84,3.26)$ & \\
\hline Sowa & $18(25.0)$ & & $2.87(I .5 I, 5.49)$ & \\
\hline Southern & $140(13.7)$ & & $1.37(0.91,2.05)$ & \\
\hline Barolong & $64(13.7)$ & & $1.37(0.87,2.15)$ & \\
\hline Ngwaketse West & $32(15.6)$ & & $1.59(0.94,2.70)$ & \\
\hline Southeast & $56(12.4)$ & & $1.22(0.77,1.93)$ & \\
\hline Kweneng East & $210(15.3)$ & & $1.56(1.05,2.32)$ & \\
\hline Kweneng West & $32(10.4)$ & & 1.00 & \\
\hline Kgatleng & $93(14.6)$ & & I.47 $(0.96,2.25)$ & \\
\hline Central-Serowe & $276(19.9)$ & & $2.14(1.45,3.16)$ & \\
\hline Central-Mahalapye & $184(17.6)$ & & $1.85(1.24,2.75)$ & \\
\hline Central-Bobonong & $112(19.5)$ & & $2.09(1.37,3.18)$ & \\
\hline Central-Boteti & $52(14.8)$ & & $1.49(0.93,2.39)$ & \\
\hline Central-Tutume & $187(19.5)$ & & $2.09(1.40,3.12)$ & \\
\hline North East & $87(21.3)$ & & $2.34(\mathrm{I} .5 \mathrm{I}, 3.6 \mathrm{I})$ & \\
\hline Ngamiland South & $104(20.1)$ & & $2.17(I .42,3.31)$ & \\
\hline Ngamiland North & $70(17.2)$ & & $1.80(1.15,2.81)$ & \\
\hline Chobe & $52(23.7)$ & & $2.69(1.66,4.34)$ & \\
\hline Ghanzi & $39(13.6)$ & & I.36 $(0.83,2.24)$ & \\
\hline Kgalagadi South & $62(18.0)$ & & $1.90(1.20,3.00)$ & \\
\hline Kgalagadi North & $26(11.7)$ & $<0.001$ & $1.14(0.66,1.97)$ & \\
\hline
\end{tabular}

Notes: *Data are expressed as number (\%) of cases that are HIV-positive; **P-value comparison across HIV-positive and -negative cases using the Chi-squared test for categorical variables; ***crude unadjusted marginal OR obtained from the standard logistic regression model and ranked from the highest to the lowest OR. Kweneng West district is taken as reference because of its low HIV prevalence in 2008. Rank of marginal OR in 2008: Selebi-Phikwe (I), Sowa (2), Francistown (3), Chobe (4), North East (5), Ngamiland South (6), Central-Serowe (7), Central-Tutume (8), Central-Bobonong (9), Kgalagadi South (I0), Orapa (II), Central-Mahalapye (I2), Ngamiland North (13), Gaborone (14), Lobatse (15), Jwaneng (16), Ngwaketse West (17), Kweneng East (18), Central-Boteti (19), Kgatleng (20), Southern (2I), Barolong (22), Ghanzi (23), Southeast (24), Kgalagadi North (25), Kweneng West (26); †Spatially adjusted posterior OR obtained from the Bayesian geoadditive regression model after controlling for nonlinear effect of age; categorical variables and the province of residence (spatial effects) (see Figure I).

Abbreviations: BAIS III, 2008 Botswana AIDS Impact Survey; OR, odds ratio; Cl, credible interval.

at HIV diagnosis and district of residence were significant risk factors for HIV in Botswana.

The estimated nonlinear effects of age at HIV diagnosis were plotted as PORs of the risk of HIV against age. Shown are the PORs together with the $95 \%$ point-wise credible intervals. There was a bell-shaped, nonlinear relationship between the risk of HIV and age. As expected, the likelihood of infection by age differed by sex, with males starting at the lowest probability, and attaining the peak later in life (40 years) than their female counterparts (peak at around 


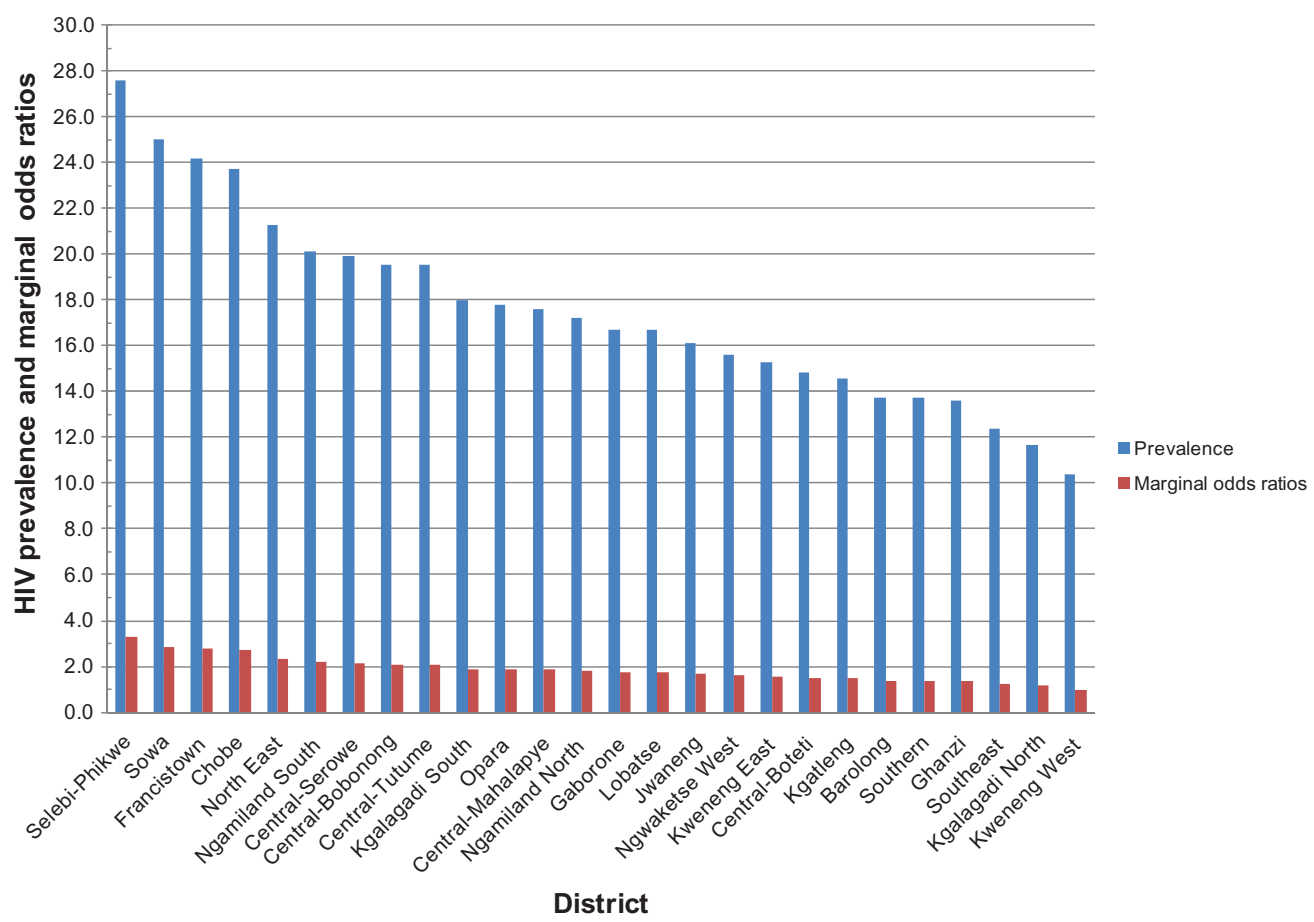

Figure I HIV prevalence by district and marginal odds ratios, Botswana 2008.

35 years old). At all other ages, the two estimates showed agreement in the increasing pattern of the probability of infection. A peak was observed between 30-40 years of age, when both males and females had the highest observed probability of HIV infection. Before age 30 , this probability increased quickly as age increased. Beyond age 40 there was a declining probability of infection, although the variation in probability increased rapidly at the same time as age continued to increase (Figure 3).

\section{Discussion}

To the best of our knowledge, this study is the first to investigate inequalities at the district level using a nationally representative household sample and to take into account the effects of both individual and area based measures of social inequalities in order to provide insight into the influences of socioeconomic, environmental, and cultural factors on HIV prevalence in Botswana.

Our findings show that HIV rates in Botswana are indeed spatially distributed. While being female and working in a relatively low status of employment were associated with increased likelihood of HIV infection, the analysis also revealed significant district specific net spatial effects of HIV that could not be explained by sex, employment status, or rural-urban location. The associations between HIV risk and different socioeconomic indicators may have different implications and causes. For example, at the individual level, people of lower employment status may not have access to protective measures and health care. Meanwhile, professional people with higher education may have increased knowledge related to health promotion and increased compliance to prevention methods - although professionals with higher income may be at higher risk of HIV through engagement in risky sexual behaviors. ${ }^{8,9}$

There was a higher proportion of males with an increase in seroprevalence at age 15 compared with females of the same age. A likely explanation is riskier intercourse among males. The percentage of adolescent males aged 15-19 years reporting condom use with their most recent sexual partner declined from $35 \%$ in 2000 to $31 \%$ in 2009 , and only $38 \%$ reported that they could access condoms on their own. ${ }^{6}$ As expected, females attained their highest peak of HIV risk 10 years earlier than males. These findings are probably due to the earlier sexual debut of males and early marriages among females compared with males. In other sub-Saharan African countries such as Zambia, it was found that respondents aged 15-19 years reporting sexual activity before age 15 were $27 \%$ in $2001 / 2002$, compared with $16 \%$ in 2007 . This decrease was probably due to HIV education. 
A

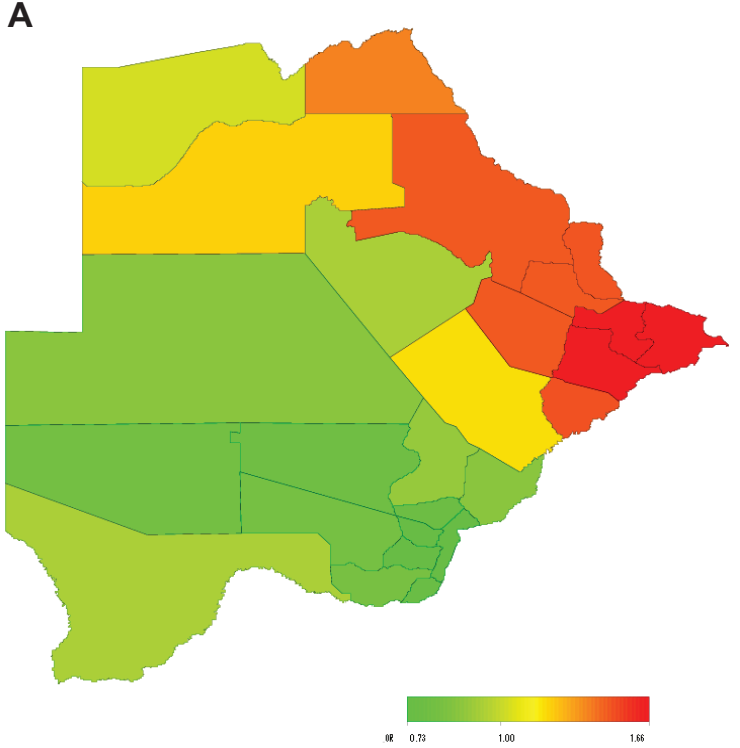

B

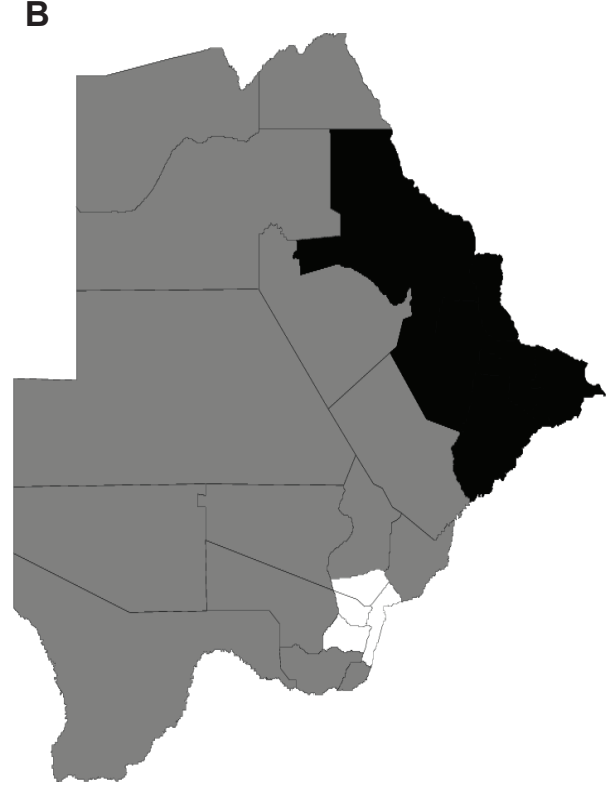

Figure 2 Total residual district spatial effects (A) and 95\% posterior probability map (B) of the risk of HIV infection in Botswana. ${ }^{3}$

Notes: (A) Red, high risk (OR: I.66); green, low risk (OR: 0.73). (B) Black, positive spatial association (high risk); white, negative spatial association (low risk); grey, no spatial association.

Abbreviations: OR, odds ratio.

While certain districts (Francistown, North East, and Selebi-Phikwe) had higher HIV prevalence, these results show that some aspect of HIV infection risk is spatial, such that districts that are in the proximity of these high prevalence districts also display elevated marginal ORs of HIV infection. Why certain districts have a higher risk of HIV infection is an area that has not been subjected to rigorous research. So while some districts are known to have a higher HIV prevalence, there is poor understanding of the contextual factors and other factors (sociocultural) that lead to these districts having a significantly higher prevalence and risk of HIV than others. However, it is well documented that geography is important at the area level. Living near a market place, for example, may be associated with increased HIV risk for young women, while living near roads connecting villages to cities increases the likelihood of migration from presumably conservative villages to more sexually permissive cities. ${ }^{10}$ Living in rural areas may be associated with poor health care including HIV testing and treatment. The district-level relationship between employment status and HIV infection may be attenuated by socioeconomic heterogeneity.

The reasons why a district like Kweneng has the lowest HIV prevalence in the country are yet to be studied and therefore understood. This gap in knowledge of sociocultural and other context-specific factors that underpin the high HIV prevalence and risk in certain districts and lower rates in other districts, points to a clear need for research to attain an understanding of the socioeconomic, cultural, and other factors that might explain the significant differentials in risk of HIV infection in Botswana.

For example, Selebi-Phikwe and Sowa, the districts with the highest HIV prevalence, are both mining towns. Mining is a predominantly male occupation, and so it is likely to draw a lot of male labor from surrounding villages and other districts in the country, resulting in a separation of spouses, thus increasing the volume of migrants into the mining areas. Studies have presented evidence of statistical association between mining and HIV risk. ${ }^{11-13}$ A study of a gold-mining community in South Africa found that miners had a significantly higher HIV prevalence rate than the wider community. ${ }^{13}$ The study also found evidence of increased prevalence of syphilis, gonorrhea, and chlamydial infection, and low condom use, all of which could increase the spread of the virus. A similar study of underground miners in South Africa found that mine workers' masculine identities render miners particularly vulnerable to HIV. ${ }^{11}$ Palmer and colleagues documented a high HIV prevalence among gold miners in Guyana, which may provide a reservoir for the virus in this region. ${ }^{12}$

Several factors may explain the relatively higher prevalence of HIV in Francistown, including the high cross-border traffic for trade and a high volume of both documented and undocumented migrants. A 2004 survey ${ }^{14}$ indicated that AIDS awareness increased in Francistown and Mahalapye compared to previous years. However, condom use was higher in southern than in northern parts of Botswana. Not surprisingly, the prevalence of sexually transmitted infections (STIs) was 

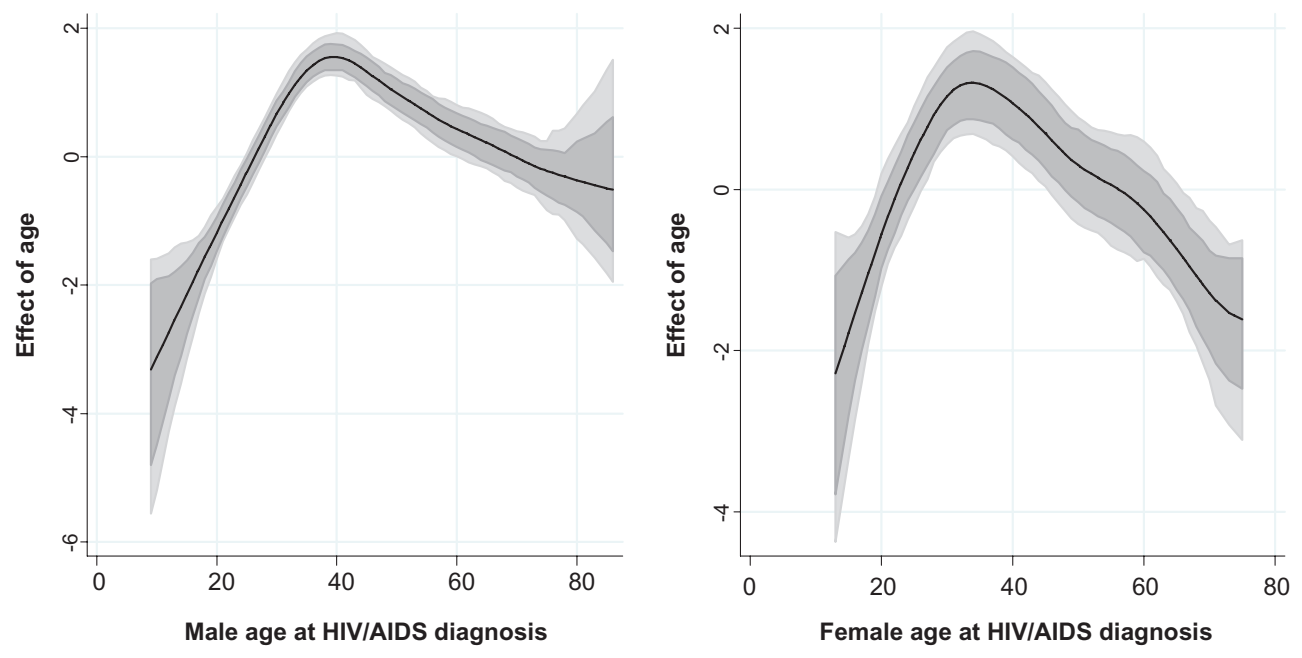

Figure 3 Sex-specific nonlinear association of the risk of HIV and age at HIV diagnosis estimated from the data. ${ }^{3}$

lower in southern than northern Botswana. However, the report mentioned that consistent condom use was generally lower with regular partners than with casual ones.

Throughout the country, there was no relationship between condom use and number of sexual partners. Subsequently, the government commissioned a study of knowledge, attitude, and practice of youths on the topic of sexual and reproductive health. ${ }^{15}$ The study revealed that almost $90 \%$ of males and females aged 15-24 years were aware of HIV, and almost all were aware of AIDS. However, while almost $90 \%$ of those aged 10-14 years were aware of AIDS (more so among females than males), less than 60\% were aware of HIV (more so among males than females). Being aware of either HIV or AIDS implies knowledge about the relationship between the two. These differences indicate that levels of awareness about HIV and AIDS are lower among adolescents than the statistics suggest. Condom use was higher among males than females, regardless of whether sexual intercourse had occurred within a regular or casual relationship. Therefore, more plausible explanations of the observed pattern of higher HIV prevalence in the north and northeast could be attributed to differences in levels of condom use, STI prevalence, knowledge of HIV, and unmeasured factors that have geographic/spatial structures.

There is need for further research in order to define the factors that explain the high infection rates in some parts of Botswana. Smaller internal studies ${ }^{16}$ have indicated evidence of lower rates of condom use and higher prevalence of STIs in the northern part of the country compared to the south. Other possible explanations for the relatively higher HIV prevalence and risk in districts along the east and northern parts of the country have centered on the proximity of such districts to countries such as Zimbabwe (to the east and northeast) and Zambia (to the north), where the HIV epidemic was first established before spreading to Botswana. The existence of major trucking routes such as Kazungula for access between southern and central Africa contributes further to the problem. This study therefore suggests a geospatial effect of HIV risk and spread across national boundaries.

Throughout the world, most HIV/AIDS prevention, treatment, and care programs have been managed with increasingly limited budgets, making it necessary for the limited resources to be deployed optimally and efficiently. A thorough understanding of the district-specific net spatial effects of HIV/AIDS can ensure that programs and interventions can achieve the greatest impact by focusing the limited resources in areas where there is the greatest risk of HIV infection.

Our findings indicate that public health interventions and health promotion for HIV prevention should take into account both individual and area variation. Planning and applying intervention measures could have different outcomes in terms of effectiveness in areas with a high degree of variability. Homogeneous policy intervention strategies may not give the required outcomes as suggested by large significant inequalities in HIV prevalence in our study.

It is worth mentioning some of the strengths and limitations of our study. The major strength is the use of a nationally representative household survey to investigate and explain district level inequalities in HIV rates using a novel approach that accounts simultaneously for individual, household, and area factors.

The main limitation of this study is the cross-sectional nature of the BAIS III data, which does not permit one to draw 
causal association between HIV prevalence and associated risk factors. Moreover, there is a time lag between exposure and detection of HIV infection. Therefore, an indicator such as employment status, which refers to the same period in which HIV was diagnosed, may not inform on exposures to HIV occurrence in earlier years.

The use of geographic area (district) as a single measure of area employment status is unlikely to provide a full assessment of area characteristics. Other factors that have not been included in the model may also explain inequalities in HIV prevalence (eg, environmental, socioeconomic, social and cultural factors, and access to health facilities and care). Finally, individual level measures of socioeconomic position, for instance, are not interchangeable in their relation to health outcomes and district-level factors.

\section{Conclusion}

This study is an extensive investigation of geographic variations of HIV in Botswana. Although inequalities in HIV prevalence have been reported in Botswana using sentinel antenatal care data, based on a large nationally representative population cross-sectional household survey, our study shows a clear significant geographic pattern of the epidemic noting individual (ie, age at diagnosis, sex, and employment status), area, and household factors (place of residence). The highest prevalence of infection was observed in the northeastern districts including Francistown and Selebi-Phikwe, but not Sowa district, while the prevalence of HIV in Gaborone remained significantly below the national average. Our study provides age- and location-adjusted prevalence maps that could be used for targeting HIV educational programs and efficient allocation of resources to higher-risk groups.

There is need for further research to unearth the social, cultural, economic, behavioral, and other factors that might explain the high infection rates in high-risk areas of Botswana. Therefore, further work is required using longitudinal studies to examine more closely the sociodemographic, behavioral, psychosocial, and distal factors (geographic location) as causal determinants of the risk of HIV/AIDS. In this regard, mixed methods using both quantitative and qualitative methods can be used to provide in-depth insights into the spatial dimension of the risk of HIV/AIDS.

\section{Disclosure}

The authors report no conflicts of interest in this work.

\section{References}

1. UNAIDS. UNAIDS report on the global AIDS epidemic 2010 - The global reference book on the AIDS epidemic and response. UNAIDS; 2010. Available from: http://www.unaids.org/globalreport. Accessed 15 January, 2012.

2. Population Reference Bureau. 2010 world population data sheet. Population Reference Bureau: Washington, DC; 2010.

3. Central Statistics Office (CSO). 2008 statistics brief, Botswana AIDS Impact Survey - preliminary results. Ministry of Health: Gaborone, Botswana; 2008.

4. Gardner J, Harrington T. Putting health on the map. American City \& County. 2003;118(11):30-34.

5. Fahrmeir L, Lang S. Bayesian inference for generalized additive mixed models based on Markov random field priors. Appl Stat. 2001;50: 201-220.

6. Kandala NB, Ji C, Cappuccio PF, Stones, NW. The epidemiology of HIV infection in Zambia. AIDS Care. 2008;20(7):812-819.

7. Kandala NB, Brodish P, Buckner B, Foster S, Madise N. Millennium development goal 6 and HIV infection in Zambia: what can we learn from successive household surveys? AIDS. 2011;25(1):95-106.

8. Shelton JD, Cassell MM, Adetunji J. Is poverty or wealth at the root of HIV? Lancet. 2005;366(9491):1057-1058.

9. Fenton L. Preventing HIV/AIDS through poverty reduction: the only sustainable solution? Lancet. 2004;364(9440):1186-1187.

10. Gabrysch S, Edwards T, Glynn JR. The role of context: neighbourhood characteristics strongly influence HIV risk in young women in Ndola, Zambia. Trop Med Int Health. 2008;13(2):162-170.

11. Campbell CK. Migrancy, masculine identities and AIDS: the psychosocial context of HIV transmission on the South African gold mines. Soc Sci Med. 1997;45(2):273-281.

12. Palmer CJ, Validum L, Loeffke B, et al. HIV prevalence in a gold mining camp in the Amazon region, Guyana. Emerg Infect Dis. 2002;8(3): 330-331.

13. Williams B, Taljaard D, Campbell C, et al. Changing patterns of knowledge, reported behaviour and sexually transmitted infections in a South African gold mining community. AIDS. 2003;17(14): 2099-2107.

14. Central Statistic Office (CSO). 2004 Botswana AIDS Impact Survey II, preliminary findings. Ministry of Health: Gaborone, Botswana; 2004.

15. Botswana, UNICEF, African Youth Alliance of Botswana, Joint United Nations Programme on HIV/AIDS, Population Services International. Baseline Study on Knowledge, Attitudes, Behaviours and Practices of Adolescents and Youth on Reproductive and Sexual Health. Gaborone, Botswana: SIAPAC; 2001.

16. Campbell EK, Rakgoasi SD. Condom use among youths in Botswana in the era of HIV and AIDS. Soc Dev Iss. 2002;24(1):56-67.
HIV/AIDS - Research and Palliative Care

\section{Publish your work in this journal}

HIV/AIDS - Research and Palliative Care is an international, peerreviewed open-access journal focusing on advances in research in HIV, its clinical progression and management options including antiviral treatment, palliative care and public healthcare policies to control viral spread. The journal welcomes original research, basic science,

\section{Dovepress}

clinical \& epidemiological studies, reviews \& evaluations, expert opinion \& commentary, case reports \& extended reports. The manuscript management system is completely online and includes a very quick and fair peer-review system. Visit http://www.dovepress.com/ testimonials.php to read real quotes from published authors. 